$63^{\text {ème }}$ Congrès de la SFCO, 03018 (2015)

DOI:10.1051/sfco/20156303018

(C) Owned by the authors, published by EDP Sciences, 2015

\title{
Evaluation qualitative des représentations de la chirurgie implantaire guidée auprès de praticiens
}

\author{
Dutreix L, Denost H, Arrivé E, Catros S \\ Faculté d'odontologie Bordeaux 2 16, 20 cours de la Marne, 33000 Bordeaux
}

Introduction : La chirurgie implantaire guidée statique (CIGS) s'est développée depuis une dizaine d'années grâce au progrès de l'imagerie tridimensionnelle et de l'informatique. La CIGS permet de simuler le positionnement optimal de l'implant dans les trois dimensions selon tout d'abord le projet prothétique final, mais aussi en fonction du volume osseux disponible et des éléments anatomiques nobles (Jung 2009). Toutefois, les complications éventuelles et le coût associé à cette technique pourraient limiter ses indications et sa large diffusion auprès des praticiens (Davarpanah 2011). L'objectif de ce travail était d'évaluer les représentations des bénéfices et des limites de la chirurgie guidée auprès de praticiens pratiquant la chirurgie implantaire.

Matériels et Méthodes : Les représentations de la CIGS par les chirurgiens dentistes ont été évaluées grâce à une étude qualitative menée auprès de praticiens pratiquant la chirurgie implantaire. Des entretiens semi-structurés ont été effectués auprès de praticiens répartis en 6 groupes en fonction de leur expérience en implantologie et de l'utilisation ou non de la CIGS. Des guides d'entretien ont été élaborés à partir de thèmes pré-établis. Les entretiens individuels ont été enregistrés afin de faciliter leur analyse qui s'est fait dans le respect de l'anonymat. La transcription et l'exploitation des entretiens ont permis de repérer des thèmes récurrents qui ont été regroupés par catégories au travers de grilles d'analyse. La première grille d'analyse a ainsi permis d'élaborer une liste de facteurs favorables et de freins à l'utilisation de la CIGS. La deuxième grille d'analyse exposait les conditions d'appropriation de cette technique par les praticiens l'utilisant.

Résultats et Discussion : La volonté d'intégrer la CIGS dans sa pratique dépend de la capacité à investir la technique, du mode d'exercice adopté par le praticien et de la volonté à se former à une nouvelle technique. Dans l'avenir, la CIGS demande à être simplifiée et optimisée au travers d'évolutions numériques et radiologiques, mais aussi d'être rendue accessible par une diminution de son coût et par davantage de formations professionnelles. En dehors de certaines limites cliniques, l'utilisation de la CIGS répond à la recherche de sécurité, de simplification et de précision en implantologie. Pour autant, le CIGS n'est pas une technique simple. Elle semble être indiquée essentiellement pour des cas complexes, réalisés par des praticiens expérimentés. L’expérience du praticien en CIGS permet ainsi de placer les implants de manière plus précise (Cushen 2013). Concernant la méthode qualitative utilisée, certaines limites existent avec notamment la vérification de la crédibilité des hypothèses générées par l'étude (Côté 2002). Les conclusions de ce travail ouvrent la porte vers des études quantitatives qui auront pour objectif de tester la véracité des hypothèses formulées.

This is an Open Access article distributed under the terms of the Creative Commons Attribution License 4.0, which permits unrestricted use, distribution, and reproduction in any medium, provided the original work is properly cited. 
Conclusion : La CIGS est déjà considérée comme une méthode très utile dans certaines situations cliniques. Le développement continu de logiciels de planification, les progrès permanents de l'imagerie numérique et la démocratisation des imprimantes 3D font probablement de cette technique une thérapeutique d'avenir.

Nom et adresse du conférencier

\section{Louise DUTREIX}

Faculté d'odontologie Bordeaux 2

16, 20 cours de la Marne

33000 Bordeaux (France)

louise.dtx@gmail.com 